\title{
Editor's choice: recent research highlights from the International Journal of Nanomedicine
}

\author{
This article was published in the following Dove Press journal: \\ International Journal of Nanomedicine \\ 19 June 2017 \\ Number of times this article has been viewed
}

\author{
Farooq A Shiekh' \\ Abdul-Rahman $M$ \\ Abu-Izzah ${ }^{2}$ \\ Vivian J Lee ${ }^{2}$ \\ Syed Mudassar' \\ 'Department of Clinical Biochemistry, \\ Sher-I-Kashmir Institute of Medical \\ Sciences (SKIMS), Srinagar, India; \\ ${ }^{2}$ Department of Basic Medical \\ Sciences, Avalon University School of \\ Medicine, Curacao, the Netherlands
}

\section{Is nanomedicine really less harmful?}

Evaluation of: Thakkar A, Chenreddy S, Thio A, Khamas W, Wang J, Prabhu S. Preclinical systemic toxicity evaluation of chitosan-solid lipid nanoparticle-encapsulated aspirin and curcumin in combination with free sulforaphane in BALB/c mice. Int $J$ Nanomedicine. 2016;11:3265-3276.

Nanomedicine ${ }^{1}$ has increasingly received a tremendous attention over the past two decades as a potential multidimensional field, developing nano-applications that are transforming a host of medical products and services, ${ }^{2,3}$ including drug delivery ${ }^{4}$ and health-monitoring devices, and the possibility of gaining new insights about "undruggable targets" and treatment through atomic-scale precision is increasing rapidly. ${ }^{5}$ Although it is uncertain as to which of the new delivery platforms will become the most effective and useful, it is certain that many new approaches will be investigated in the years to come., ${ }^{4,6}$

In one of the recent issues of the International Journal of Nanomedicine, Thakur et al investigated the systemic toxicity of nanoengineered chitosan-solid lipid particles encapsulated with aspirin and curcumin in combination with free sulforaphane (ACS c-SLNs) in BALB/c mice, which is a very elaborative study using an animal model with the capability to address how the benefits of new drug delivery approaches could be achieved while minimizing potential risks. However, many toxicologists argue that commercialization of nanomaterials is rapidly overtaking efforts to study their impact on human and environmental health, and mostly, the toxicity of these particles remains unclear. ${ }^{7}$ For a therapeutic molecule to be successful, it must behave properly in physiological conditions, in addition to interacting with its molecular target, and should undergo clinical trials where we can learn whether the mechanistic ideas are having a therapeutic benefit and what the drawbacks are in terms of side effects. ${ }^{8}$

Noticeably, based on the findings of this study, the authors could not identify any signs of toxicity in acute, subacute, and subchronic examinations following oral administration of ACS c-SLNs, which clearly indicates that the oral dosing regimens were safe at the levels tested for a long-term examination to prevent the onset of pancreatic cancer. Importantly, the engineering of such carriers would further enable researchers to improve their design to form advanced "delivery platforms" that accompany an escort intelligent enough to evade destruction and minimize toxicity. ${ }^{9}$ It is likely that we will be able to make those smart artificial machines or vehicles at the nanoscale, which could be used, for example, to develop new types of computers, do finely targeted drug delivery, or carry out computations inside the body for diagnostic evaluations with fewer side effects. ${ }^{10}$
Correspondence: Farooq A Shiekh Department of Clinical Biochemistry, Sher-I-Kashmir Institute of Medical Sciences (SKIMS), Srinagar I900I I, India Tel +91 1942401013

Fax +91 1942403470

Email shiekh.fa@gmail.com 
In summary, understanding and preventing risk often has a low priority in the competitive world of research funding, and embracing a fixed idea is one of the main dangers in the evolution of any scientific discipline. Ideally, errors or drawbacks must be uncovered in the trial by fire of rigorous testing using cutting-edge molecular tools or animal models, and the safety agencies around the world should continue efforts to work out how best to regulate these novel materials. These are very important concerns. ${ }^{11}$ However, it is going to be very hard to come up with a nanotherapeutic molecule that will be more toxic than conventional drugs out in the market. If true, nanomedicine will at least be less harmful than today's cancer fighters, but if it works as intended, it must also prove far more effective.

\section{Combination nanotherapeutics: a real promise}

Evaluation of: Jun X, Zou B, Luo L, et al. Codelivery of thioridazine and doxorubicin using nanoparticles for effective breast cancer therapy. Int J Nanomedicine. 2016;11: 4545-4552.

Breast cancer is a devastating disease typically riddled with genetic mutations, a leading cause of death afflicting millions of women worldwide. ${ }^{12}$ Although emerging genome-analysis methods are now sufficiently powerful, fast, and reliable that they are underpinning efforts to elucidate the molecular mechanics of human cancers, that could prove to be helpful for target validation and molecular therapeutic interventions, unfortunately, our basic approach to treat cancer has remained essentially unchanged over decades and the medicines used have clear limitations. ${ }^{13,14}$ Nevertheless, in the quest for better drug delivery, nanomedicine represents a new "powerful platform", which holds a great promise and is increasingly gaining momentum, ${ }^{15}$ to deliver multiple drugs ${ }^{16}$ at a time, which is profoundly an "advancing approach" to treat cancer patients.

A new study by Jun et al describes the development of an emerging multidrug-based combination nanotherapeutic approach using methoxypoly (ethylene glycol)-poly(Llactic acid) nanoparticles, an innovative design with the potential to address one of the current radical understanding about the future cancer therapy, how to get enough of the right combination of multiple drugs to the right target. The fundamental aim has got to be to hit multiple targets simultaneously ${ }^{6}$ so that the tumors cannot develop resistance. These newly emerging approaches ideally seek to completely eliminate highly complex tumor tissues, that have, thus far, been resistant to available therapies, not only by the elimination of every malignant cell type but essentially by confronting cancer at different molecular levels or pathways involving tumor cell growth, migration, and invasion, at all possible targets in the most effective manner. ${ }^{17}$

In the $2020 \mathrm{~s},{ }^{1,18}$ nanomedicine will most likely see continued growth in the discovery and development of new combination therapeutic approaches with regard to the existing drugs, targeting multiple genetic pathways and attacking specific attributes of each disease using multidrug delivery devices. However, the future of oncology - and the opportunity to eliminate the suffering and death due to cancer - will absolutely depend on our ability to confront cancer at its molecular level. ${ }^{19}$

Taken together, presently both oncologists and academic researchers are trying very hard to figure out how to make smart combination therapies that really work. This push toward most effective treatment has been underway for many years, but there should also be an effort to combine all of these advances, engineering clinically relevant delivery platforms with a capability of carrying potent multiple drugs designed to kill "only" cancer cell types. The promise of these advances in human cancer remains quite real, and with ever promising results from the clinic, humankind will be on the verge of gaining immense, new power to heal.

\section{Next-generation nanodevices}

Evaluation of: Zheng H, Li X, Chen C, et al. Quantum dotbased immunofluorescent imaging and quantitative detection of TOP2A and prognostic value in triple-negative breast cancer. Int J Nanomedicine. 2016;11:5519-5529.

Emergence of new effective imaging devices for early diagnosis and treatment continues to become an inevitable need and indispensable scope for both oncologists and academic researchers wishing to study molecular complexities of cancer with an astonishing level of detail. ${ }^{20,21}$ Quantum dots (QDs), the luminescent size- and shape-tunable nanocrystals, are also still a research frontier, ${ }^{22}$ with the potential of singlecell molecular profiling ${ }^{23}$ in systems biology, gene expression studies, signaling pathway analysis, and molecular diagnosis; ${ }^{24}$ however, QDs are next-generation nanodevices that would open up application, in which nanoparticles can be injected into a tumor, for example, to make it glow and help surgeons to remove all traces of it. ${ }^{25}$

Recently, Zheng et al for the first time investigated the relationship between TOP2A protein expression and major clinical pathological parameters using QD-based immunofluorescent imaging and quantitative analytical system in triple-negative breast cancer. In recent years, biotechnology and biomedical research have immensely benefited from the introduction of a variety of sophisticated imaging tools whose well-defined, optically distinguishable signatures enable simultaneous 
tracking of numerous clinically important indicators. Notably, outstanding QD photostability proves essential for robust image acquisition and quantitative analysis of staining intensity, which is otherwise a fundamental limitation of other organic molecular probes. ${ }^{2,25}$ Such advances in QD synthesis and surface nanofabrications achieved during the last decade have produced multicolor QD-antibody conjugates aiming to expand multiplexing capabilities of immunofluorescence staining, offering exciting opportunities in gene expression studies.

In addition to sophisticated cell-based and in vivo tests, promising candidate drugs have to be eventually tested in various animal models because even a smaller subset of target interactions could affect tumor development and progression in vivo. ${ }^{26}$ It is therefore pathophysiologically challenging to identify functionally relevant target genes and pathways on the basis of dysregulated gene expression profiles in tumor cell types. Common approaches to study target genes and function in cancer involve experimental complexities of clinically important gene expression evaluation in cell lines and mouse models. ${ }^{27,28}$ Although such models have yielded significant mechanistic molecular insights into cancer biology, they are not fully capable of capturing those molecular complexities of tumorigenesis in patients. However, nanoprobes, such as QDs, are an advancing imaging system that could fluoresce in a wide variety of colors and work as cell spies, showing the movements of their molecular quarries and comprehensively enabling the investigator to follow the molecular events on a camera with an astonishing level of detail.

These advancing molecular tools not only have the potential to significantly help academic researchers to "better visualize" and understand how a therapeutic molecule reacts, at a molecular level, in vivo, but also would embrace a translational approach to epigenetics, to determine the abnormal epigenetic patterns found in tumors and the use of epigenetic markers to predict which cancer patients will respond to an anticancer drug that blocks DNA methylation or carry out computations inside the body for diagnostic purposes.

\section{Acknowledgments}

The authors would like to acknowledge Dr Kaisar and Dr Aaliya, Department of Clinical Biochemistry, SKIMS, for their valuable comments.

\section{Disclosure}

The authors report no conflicts of interest in this work.

\section{References}

1. Dresselhaus MS. A revolution of nanoscale dimensions. Nat Rev Mater. 2016;1(1):15017.
2. Roberts J, Bagci IE, Zawawi MA, et al. Using quantum confinement to uniquely identify devices. Sci Rep. 2015;5:16456.

3. Smith C. Tools for drug discovery: tools of the trade. Nature. 2007; 446(7132):219-222.

4. Shiekh FA. Highlights from recent advances in nanomedicine Nanomedicine (Lond). 2014;9(9):1287-1289.

5. Rolle AM, Hasenberg M, Thornton CR, et al. ImmunoPET/MR imaging allows specific detection of Aspergillus fumigatus lung infection in vivo. Proc Natl Acad Sci U S A. 2016;113(8):E1026-E1033.

6. Shiekh FA, Mian SH, Arja SB, Raghavendra Rao MV. Targeted combination nanotherapeutics in cancer a real promise. Nanomedicine (Lond). 2015;10(12):1855-1857.

7. Service RF. Nanotechnology. Calls rise for more research on toxicology of nanomaterials. Science. 2005;310(5754):1609.

8. Gibbs JB. Mechanism-based target identification and drug discovery in cancer research. Science. 2000;287(5460):1969-1973.

9. Shiekh FA, Farooq O, Mian SH, Bautista RL, Arja SB, Andrabi KI. The pitfalls of growing nanomaterials. Nanomedicine (Lond). 2016; 11(13):1635-1638.

10. Khodadoust MS, Olsson N, Wagar LE, et al. Antigen presentation profiling reveals recognition of lymphoma immunoglobulin neoantigens. Nature. 2017;543(7647):723-727.

11. Riego-Sintes J. Regulation: safety-test initiatives for nanomaterials. Nature. 2012;482(7383):35.

12. Keckesova Z, Donaher JL, De Cock J, et al. LACTB is a tumour suppressor that modulates lipid metabolism and cell state. Nature. 2017;543(7647):681-686.

13. Shiekh FA. Personalized nanomedicine: future medicine for cancer treatment. Int J Nanomedicine. 2013;8:201-202.

14. Ryan RJ, Bernstein BE. Molecular biology. Genetic events that shape the cancer epigenome. Science. 2012;336(6088):1513-1514.

15. Dong Y, Love KT, Dorkin JR, et al. Lipopeptide nanoparticles for potent and selective siRNA delivery in rodents and nonhuman primates. Proc Natl Acad Sci U S A. 2014;111(11):3955-3960.

16. Aires A, Ocampo SM, Simões BM, et al. Multifunctionalized iron oxide nanoparticles for selective drug delivery to CD44-positive cancer cells. Nanotechnology. 2016;27(6):065103.

17. Shiekh FA. Targeted nanotherapeutics in cancer. Int J Nanomedicine. 2014;9:1627-1628.

18. Pisano GP. Drug Discovery. Nature. 2010;463:26-32.

19. Jain RK, Stylianopoulos T. Delivering nanomedicine to solid tumors. Nat Rev Clin Oncol. 2010;7(11):653-664.

20. Yoon HJ, Kozminsky M, Nagrath S. Emerging role of nanomaterials in circulating tumor cell isolation and analysis. ACS Nano. 2014; 8(3):1995-2017.

21. Takahashi Y, Shevchuk AI, Novak P, et al. Topographical and electrochemical nanoscale imaging of living cells using voltage-switching mode scanning electrochemical microscopy. Proc Natl Acad Sci USA. 2012;109(29):11540-11545.

22. Liu X, Ji X, Liu M, et al. High-performance Ge quantum dot decorated graphene/zinc-oxide heterostructure infrared photodetector. ACS Appl Mater Interfaces. 2015;7(4):2452-2458.

23. Tang R, Xue J, Xu B, Shen D, Sudlow GP, Achilefu S. Tunable ultrasmall visible-to-extended near-infrared emitting silver sulfide quantum dots for integrin-targeted cancer imaging. ACS Nano. 2015; 9(1):220-230.

24. Mian SH, Patel NA, Shah F, Arja SB, Shiekh FA. Research highlights from the International Journal of Nanomedicine 2014. Int $J$ Nanomedicine. 2015;10:2503-2505.

25. Lim X. The nanoscale rainbow. Nature. 2016;531(7592):26-28.

26. Pu K, Chattopadhyay N, Rao J. Recent advances of semiconducting polymer nanoparticles in in vivo molecular imaging. J Control Release. 2016; 240:312-322.

27. Lienert F, Lohmueller JJ, Garg A, Silver PA. Synthetic biology in mammalian cells: next generation research tools and therapeutics. Nat Rev Mol Cell Biol. 2014;15(2):95-107.

28. Shiekh FA. Synthetic nanocarriers as a new paradigm. Nanomedicine (Lond). 2014;9(15):2259-2262. 
Dove Medical Press encourages responsible, free and frank academic debate. The content of the International Journal of Nanomedicine 'Editorial' section does not necessarily represent the views of Dove Medical Press, its officers, agents, employees, related entities or the International Journal of Nanomedicine editors. While all reasonable steps have been taken to confirm the content of each Editorial, Dove Medical Press accepts no liability in respect of the content of any Editorial, nor is it responsible for the content and accuracy of any Editorial.

International Journal of Nanomedicine

Dovepress

\section{Publish your work in this journal}

The International Journal of Nanomedicine is an international, peerreviewed journal focusing on the application of nanotechnology in diagnostics, therapeutics, and drug delivery systems throughout the biomedical field. This journal is indexed on PubMed Central, MedLine, CAS, SciSearch ${ }^{\circledR}$, Current Contents $₫ /$ Clinical Medicine, Elsevier Bibliographic databases. The manuscript management system is completely online and includes a very quick and fair peer-review system, which is all easy to use. Visit http://www.dovepress.com/ testimonials.php to read real quotes from published authors.

Submit your manuscript here: http://www.dovepress.com/international-journal-of-nanomedicine-journal 